\title{
A Case Of Pneumoperitoneum In A Patient With Blunt Injury Thorax
}

\author{
*Dr.Prabhakaran. ${ }^{1}$, Dr.Rajeev Thilak $C^{2}$, Dr.Ameesh Isath ${ }^{2}$, Dr.Ashitha Pathrose ${ }^{3}$. \\ ${ }_{1,2,3}$ (institue of general surgery, madras medical college, chennai,india ${ }^{l}$ \\ Corresponding author: *Dr.Prabhakaran.U
}

\begin{abstract}
Pneumoperitoneum in traumatic patients almost always indicates a underlying bowel pathology. We present a case report where pneumothorax and pneumomediastinum associated with Tracheobronchial injury causing/presenting as pneumoperitoneum. Treatment Protocol of such presentation has not yet been standardised due to its rare presentation.
\end{abstract}

Keywords : pneumoperitoneum, pneumothorax, pneumomediastinum, tracheobronchial injury

\section{Introduction}

Traumatic tracheobronchial injuries can present in polytrauma patients which presents clinically as dyspnea and will be associated with Pneumomediastinum (72\%), Pneumothortax (7\%), subcutaneous emphysema $(38 \%)^{(1)}$. Pnemoperitoneum may occur along with pneumothorax and pneumomediastinum. In such presentation, the presence of bowel injury as a etiology causing pneumoperitoneum without free fluid is small (2). Though the presence of bowel injury can't be ruled out based only on the imaging studies, severe tracheobronchial injury causing pneumoperitoneum without free fluid, if present, has to be considered. We present such a presentation where tracheobronchial injury causing pneumoperitoneum, abdominal etiology been ruled out by laparotomy.

\section{Case Report:}

We received a young male of age 24 , in our emergency department with with polytrauma following road traffic accident. He was intubated due to low Glascow Coma Scale (3/15). Primary survey suggested presence of traumatic brain injury with equal air entry and satisfying $\mathrm{O} 2$ saturation. Abdominal examination showed, no guarding,no rigidity and was normal. CT Imaging showed left Frontal lobe and brain stem contusion with no significant thoracic and abdominal pathology on the initial time. Patient was observed during which he developed subcutaneous emphysenma, drop in saturation and abdominal distension. Clinically there was pneumothorax and pneumomediastinum. Imagings were repeated which showed Bilateral pnemothorax, pneumomediastinum, right lung contusion and pneumoperitoneum without any intra-abdomial free fluid. Tube thorocotomy was done bilaterally and to rule out any bowel pathology we proceeded with laparotomy which showed normal bowel and solid organs. The patient could not be saved due to his major neurological injury.

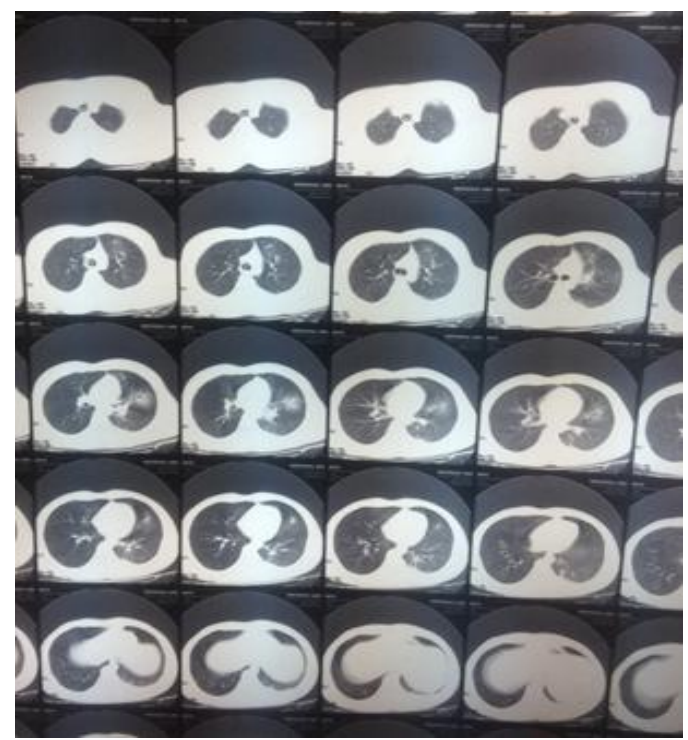




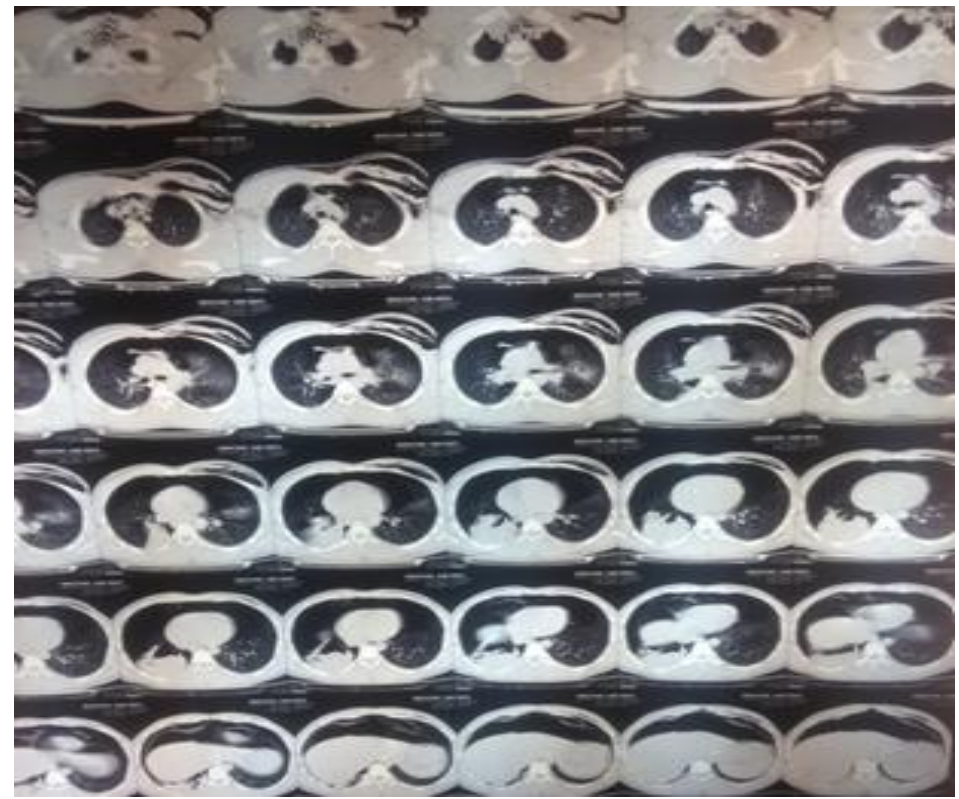

Initial CT CHEST- CT CHEST showing pneumothorax and pneumomediastinum
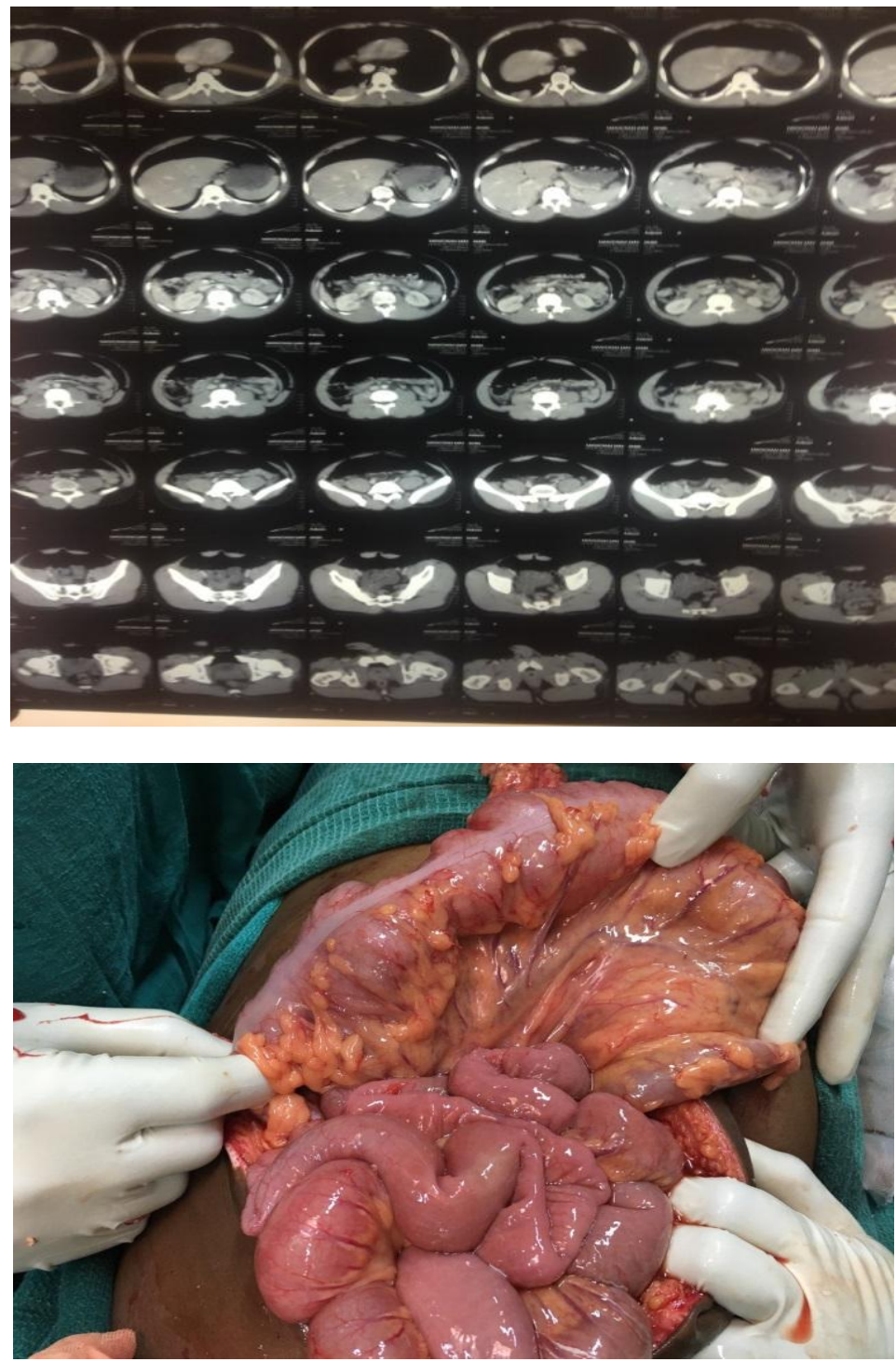

CT ABDOMEN showing pneumoperitoneum Laprotomy - normal 


\section{Discussion}

The present case describes a rare case with pneumoperitoneum after traumatic blunt injury. The association between pneumoperitoneum and pnemothorax is considered rare ${ }^{(3)}$. Traumatic pneumomediastinum with pneumoperitoneum can occur in $10 \%$ of blunt injury chest. In more than $95 \%$ it happens due to air leak from rupnemothoraxured alveoli into the interstitial space ${ }^{(3)}$.CT abdomen in our patient showed air along the falciform ligament which could be another possible way of transmission of air from thoracic cavity to abdomen.Based on certain experimental studies, it has been said that interstitial emphysema develops when intratracheal pressure exceeds $40 \mathrm{~cm}$ of water, pneumomediastinum develops at $50 \mathrm{~cm}$ of water, pneumoperitoneum can occur when the pressure exceeds $60 \mathrm{~cm}$ of water ${ }^{(4)}$. As the intrathoracic pressure rises the air dissects along the fascial planes/sheaths of adjacent vessels into the mediastinum. The air can then dissect into various spaces including the pleural and along the esophagus and thoracic great vessels into the retroperitoneum, where it may rupnemothoraxure into the peritoneal cavity ${ }^{(5)}$. Rupnemothoraxure of diaphragm can also be another, though rare, cause of pneumoperitoneum ${ }^{(6)}$."A rupnemothoraxured abdominal viscus may occur along wit pnemothorax in some cases ${ }^{(2)}$. There is also a possibility that pneumoperitoneum can occur in such patients following visceral perforation permitting only air not the bowel contents ${ }^{(7)}$. There are two issues to be sorted out in case of CT-detected pneumoperitoneum, the source of the free air and the management of the same."

'In the study by Kane and colleagues, approximately $80 \%$ of blunt trauma patients with CT-detected pneumoperitoneum did not have an abdominal injury requiring surgery; however, the series in this study was too small to draw a solid recommendation. CT detected pneumoperitoneum warrants further larger studies to intervene surgically. The classic CT findings that mandate surgical exploration include free peritoneal fluid and mesenteric or bowel wall thickening ${ }^{(8)}$. Recently, Marek et al ${ }^{(9)}(2014)$ evaluated 78 cases and concluded that CT scans may detect free air that is not always clinically significant."

"Hefny et al. ${ }^{(10)}$ (2014) showed that free intraperitoneal air found on abdominal CT scan of blunt trauma patients (21 cases) was an unreliable radiological finding for bowel perforation and the decision for laparotomy should be based on combined clinical and radiological findings. They suggested active observation,with conservative management that may avoid unnecessary laparotomy. Since the study is retrospective with small sample size solid conclusions could not be made.".

''In our case,since the patient was sick and clinically detoriating and the amount of pneumoperitoneum was enormous and it was clearly very difficult to rule out bowel injury, so we decided to go ahead with exploratory laparotomy for definite diagnosis. We found no evidence of any bowel injury. We believe it needs further evidence to carry out conservative line of management for these kind of clinical scenarios".

\section{Conclusion}

In a case of blunt injury thorax with pneumothorax and concomitant pneumoperitoneum, exploratory laparotomy sould be done to rule out bowel injury. Some selected patients can still be managed conservatively, since there are no controlled trials to provide proper management protocols, decision have to be made cautiously with close observation. More prospective studies are warranted before considering conservative management.

\section{Acknowledgement}

I would like to thank Prof.Dr.Suresh M.S for his immense support and guidance.

I would like to thank my institute, IGS, Madras Medical College for their support.

Finally my colleagues, Dr.Ameesh Isath and Dr.Ashitha Pathrose for their valuable assistance.

\section{References}

[1]. Schneider T., Storz K., Dienemann H., Hoffmann H. Management of iatrogenic tracheobronchial injuries: a retrospective analysis of 29 cases. Ann Thorac Surg. 2007;83:1960-1964.

[2]. Glauser F.L., Bartlett R.H. Pneumoperitoneum in association with pneumothorax. Chest. 1974;66:536-540.

[3]. Nayak S.R., Anindita M., Soren D.K., Nagendra S.B. Spontaneous pnemoperitoneum after blunt trauma chest - diagnostic dilemma-role of diagnostic laparoscopy - case report. J Trauma Treat. 2013;2:164.

[4]. Grosfeld J.L., Boger D., Clatworthy H.W., Jr. Hemodynamic and manometric observations in experimental air-block syndrome. J Pediatr Surg. 1971;6:339-344.

[5]. Assenza M., Passafiume F., Valesini L., Centonze L., Romeo V., Modin C. Pneumomediastinum and pneumoperitoneum after blunt chest trauma: the Macklin effect. J Trauma Treat. 2012;1(1):1-5.

[6]. Ferrera P.C., Chan L. Tension pneumoperitoneum caused by blunt trauma. Am J Emerg Med. 1999;17:351-353

[7]. Gantt Cb., Jr., Daniel W.W., Hallenbeck G.A. Nonsurgical pneumoperitoneum. Am J Surg. 1977;134:411-414.

[8]. Sabsovich I., Desai R., Alba R., Yunen J., Sammett D. 2014. Idiopathic pneumoperitoneum after blunt chest trauma. Available at hcplive.com/publications/surgical-rounds.2008-04-02 [accessed on July 2]

[9]. Marek A.P., Deisler R.F., Sutherland J.B., Punjabi G., Portillo A., Krook J. CT scan-detected pneumoperitoneum: an unreliable predictor of intra-abdominal injury in blunt trauma. Injury. 2014;45(1):116-121. 
[10]. Hefny A.F., Kunhivalappil F.T., Matev N., Avila N.A., Bashir M.O., Abu-Zidan F.M. Usefulness of free intraperitoneal air detected by CT scan in diagnosing bowel perforation in blunt trauma: experience from a community-based hospital. Injury. 2014;(Sepnemothoraxember 16) pii:S0020-1383(14)00431-8

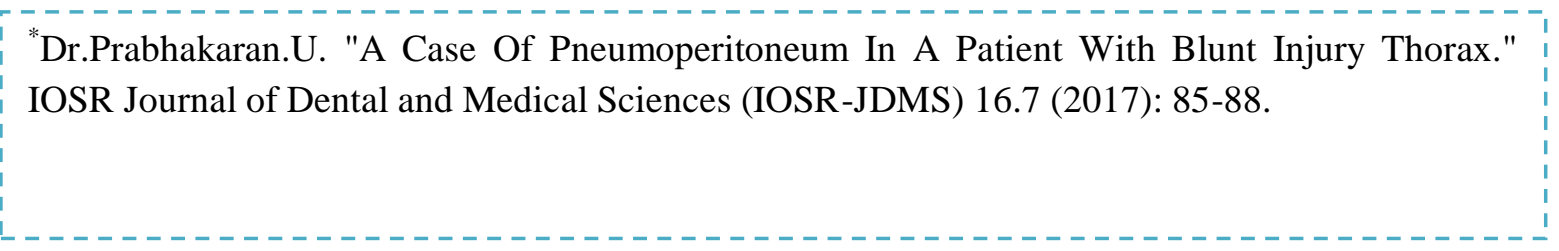

